Reprod. Nutr. Dévelop., 1987, 27 (1 B), 223-224.

\title{
Comparaison de l'oxyde de chrome et de l'ytterbium pour la mesure des flux duodénaux par simple et par double marquage chez la vache laitière
}

\author{
J. L. PEYRAUD
}

Station de Recherches sur la Vache laitière, I.N.R.A., Saint-Gilles, 35590 L'Hermitage, France.

Summary. We compared the use of chromic oxide and ytterbium to estimate duodenal flow in lactating cows fed with fresh grass. The flows of organic matter (MO) and nonammonia nitrogen (NNA) were not significantly different with chromic oxide or ytterbium when used in either a single or dual marker technique. Nonetheless, chromic oxide gave some erroneous flows because it behaved independently of the particulate matter in the digesta and during filtration in the double-marker technique.

La méthode du double marquage (Faichney, 1980) permet de corriger la différence éventuelle de composition entre l'effluent prélevé à travers une canule simple, placée à l'entrée du duodénum, et le contenu qu'il représente. Dans cette technique, l'emploi de l'oxyde de chrome a été très critiqué puisqu'il ne se fixe pas aux particules alimentaires (Faichney 1972). Nous avons comparé ici l'ytterbium et le chrome fixé sur poudre de cellulose, en simple ou en double marquage en association avec le PEG, dans la mesure des flux digestifs chez la vache laitière.

Matériel et méthodes. Quatorze bilans digestifs ont été réalisés sur 8 vaches Pie Noires fistulées du rumen et du duodénum qui ont reçu du fourrage vert 19 à $14 \mathrm{~kg} / \mathrm{j}$ de matière organique : trèfle blanc, dactyle, ray-grass anglais). L'oxyde de chrome (CR : $24 \mathrm{~g} / \mathrm{j}$ ) a été mis directement dans le rumen 3 fois par jour aux heures des repas (7 h 30-14 h 30-21 h 30). Le PEG (250 g/j) et l'ytterbium (YB : $2 \mathrm{~g} / \mathrm{j})$ ont été infusés en continu séparément. Après 5 jours d'adaptation, les fèces et l'urine ont été collectées séparément pendant 5 j (J1 à J5). Un échantillon moyen de contenu de duodénum a été constitué par 18 prélèvements effectués sur 3 jours consécutifs (J2, J3, J4). La totalité de l'échantillon moyen ainsi obtenu a été filtré et la reconstitution a été effectuée à partir du filtrat et du résidu. L'ytterbium a été dosé par spectrométrie d'absorption atomique après une minéralisation nitrique. Les flux ont été estimés à partir des quantités de marqueur récupérées dans les fèces.

Résultats et discussion. La récupération de l'ytterbium a été pratiquement complète $(0,98 \pm 0,04)$ et plus élevée que celle du chrome $(0,93 \pm 0,04)$. Celle du PEG a été plus faible et beaucoup plus variable $(0,83 \pm 0,09)$.

Les flux de matière organique (MO) et d'azote non ammoniacal (NNA) à l'entrée de l'intestin estimés par simple marquage avec le chrome ou l'ytterbium n'ont pas été significativement différents mais le chrome a donné des résultats plus variables (tabl. 1). L'écart extrême entre les 2 méthodes a été de $1,7 \mathrm{~kg} / \mathrm{j}$ de MO soit $17 \%$ de MO ingérée (MOI) et de $90 \mathrm{~g} / \mathrm{j}$ de NNA (24\% N ingéré, NI).

L'écart entre les flux de MO calculés par simple marquage avec l'ytterbium et le PEG qui traduit l'erreur d'échantillonnage, est toujours resté inférieur à $15 \%$. $L^{\prime}$ écart a été plus variable lorsque les flux ont été calculés avec le $C R$ et le PEG 
(compris entre 0 et $33 \%$ ). Les deux couples de marqueurs ne donnent donc pas toujours la même information sur la représentativité de l'échantillon prélevé.

TABL. 1. - Comparaison des flux duodénaux de matière organique (MO) et d'azote non ammoniacal (NNA) estimés par simple ou par double marquage avec l'oxyde de chrome (CR), I'ytterbium (YB) et le PEG.

\begin{tabular}{lccccc}
\hline & PEG & CR & YB & CR/PEG & YB/PEG \\
\hline Flux MO (\% MO ingérée) & $47,2(2,7)$ & $49,8(5,7)$ & $50,8(2,8)$ & $55,4(13,5)$ & $52,3(2,8)$ \\
Flux NNA (\% N ingéré) & $73,9(4,2)$ & $77,8(8,5)$ & $79,2(4,1)$ & $80,4(11,9)$ & $78,0(3,5)$ \\
\hline
\end{tabular}

( ) : Ecart type d'une moyenne.

L'écart moyen entre les flux de MO estimés par double marquage avec les couples YB/PEG et CR/PEG est faible ( $3 \%$ de $\mathrm{MOI}, 0,38 \mathrm{~kg} / \mathrm{j}$ ) mais avec une variabilité individuelle des flux plus importante avec le chrome (tabl. 1) en raison des valeurs aberrantes obtenues à 2 reprises par suite d'une correction trop forte apportée au contenu prélevé (MS corrigée : 5,49 et 7,50 avec CR/PEG vs 4,39 et $4,06 \%$ avec YB/PEG). A l'exclusion de ces 2 bilans, l'écart extrême entre les 2 couples de marqueurs est de $11,5 \%$ de $\mathrm{MOI}$ soit $1,12 \mathrm{~kg} / \mathrm{j}$. La liaison entre les flux de NNA $(g / j)$ estimés avec les couples $Y B / P E G$ et CR/PEG est très étroite (Flux CR/PEG =0,99 Flux Yb/PEG - 8, R2 $=0,97$, Syx $=15, n=12$ ).

Le double marquage conduit à des flux de NNA peu différents de ceux obtenus en faisant la moyenne des 2 flux calculés par simple marquage $(313 \mathrm{vs} 310 \mathrm{~g} / \mathrm{j}$ avec YB et PEG ; 293 vs 292 avec CR et PEG quand on exclut les 2 bilans aberrants) et il permet de réduire légèrement la variabilité des estimations avec le couple YB/PEG.

Le dosage de l'ytterbium est sensible et reproductible mais il est soumis aux interférences des minéraux contenus dans les échantillons. Si ces effets ne sont pas pris en compte, l'erreur sur les flux calculés par double marquage est en moyenne de $6 \%$ pour MO et de $3 \%$ pour NNA.

L'ytterbium doit être préféré au chrome qui, en moyenne, donne des résultats peu différents de l'ytterbium, mais conduit parfois à des flux erronés, du fait de son transit indépendant des particules dans le tube digestif et lors de la filtration réalisée dans le double marquage. 